\title{
Proteomic Analysis of the Systemic Response to Radiographic Contrast Media
}

\author{
Juan Martinez • Warren K. Laskey • Cheri Wells • \\ Armin Foghi • Sarah Rohde • Mark Ricciardi • \\ Charlotte Mobarak
}

Published online: 15 July 2010

(C) Springer Science+Business Media, LLC 2010

\begin{abstract}
Introduction Radiographic contrast media (RCM) have numerous effects on the hemostatic system, inflammatory pathways, and vascular endothelium. Given the increasing number of high-risk patients undergoing radiographic procedures, more information regarding the systemic effects of RCM is needed.

Methods Blood samples prior to baseline, 4 and $24 \mathrm{~h}$ following elective coronary angiography in 10 patients, were subjected to matrix-assisted laser desorption/ionization time-of-flight mass spectrometry. Data are presented as the ratio of the protein mass at 4 (iTRAQ4) and $24 \mathrm{~h}$ (iTRAQ24) compared to baseline. A ratio $>1.0$ and a ratio $<1.0$ indicate production and consumption, respectively, relative to baseline.

Results In this sample, we identified 102 proteins with a confidence interval of $\geq 90 \%$. Six proteins were identified at each time point in all patients. Of the proteins identified, apolipoprotein A-I, apolipoprotein A-II, complement C3, fibrinogen beta chain, immunoglobulin $\alpha$, and prothrombin revealed an iTRAQ ratio $<1.0$ at $4 \mathrm{~h}$ when compared to baseline (all with $p$ value $<0.05$ ) and a trend toward baseline levels at $24 \mathrm{~h}$.

Conclusions Systemic administration of RCM results in a variety of alterations to the proteome. Of interest, there is activation of the thrombotic and inflammatory pathways as well
\end{abstract}

J. Martinez $\cdot$ W. K. Laskey $(\bowtie) \cdot$ C. Wells $\cdot$ A. Foghi $\cdot$

M. Ricciardi

Department of Medicine-Division of Cardiology,

University of New Mexico Health Sciences Center,

Albuquerque, NM, USA

e-mail: WLaskey@salud.unm.edu

S. Rohde $\cdot$ C. Mobarak

Biomedical Sciences-Neurosciences Department, University of New Mexico Health Sciences Center, Albuquerque, NM, USA as an interaction with lipoprotein metabolism. These changes are most pronounced at $4 \mathrm{~h}$ but may persist through $24 \mathrm{~h}$ and may be of clinical relevance in patients at risk for thromboticand inflammatory-mediated consequences of atherosclerosis.

Keywords Proteomics - Radiographic contrast media . Coronary angiography $\cdot$ iTRAQ labeling .

Mass spectrometry

\section{Introduction}

Use of radiographic contrast media (RCM) continues to expand in the realm of cardiovascular medicine [1, 2]. Given its essential role in diagnostic and therapeutic radiographic procedures, it is critical to add to our knowledge the effects of RCM on blood proteins and formed elements of the blood. Studies evaluating the effects of RCM on the cellular components of blood [3-8] and vascular endothelium $[9,10]$ have produced inconclusive and conflicting results with, consequently, unclear clinical relevance. Despite the overall safety profile documented by large-scale studies of contrast agents [11, 12], there continues to be controversy surrounding the proinflammatory [13-15] and pro-thrombotic potential of RCM [16-18]. The increasing number of high-risk patients undergoing diagnostic and therapeutic procedures increases the potential for higher rates of adverse effects associated with RCM. Accordingly, a broader understanding of the effects of RCM on the "proteome" is of potential clinical relevance. We conducted an exploratory feasibility study in an unselected group of patients referred for coronary angiography and, using high-throughput proteomic analysis, analyzed serum plasma prior to and following administration of RCM. 
Table 1 Baseline characteristics for all patients

\begin{tabular}{ll}
\hline Demographic factors & Patient data $(n=10)$ \\
\hline Age & $49 \pm 14$ \\
Male (\%) & $7(70)$ \\
Risk factors (\%) & \\
Chronic smoker & $4(40)$ \\
Diabetes mellitus & $4(40)$ \\
Hypercholesterolemia & $9(90)$ \\
Hypertension & $4(40)$ \\
Illicit drug use & $1(10)$ \\
Aspirin therapy (\%) & $10(100)$ \\
Statin therapy (\%) & $8(80)$ \\
Chronic kidney disease & 0 \\
Coronary artery disease (\%) & $9(90)$ \\
History of coronary artery bypass (\%) & $2(20)$ \\
Amount of Contrast used (ml) & $247 \pm 198$ \\
\hline
\end{tabular}

\section{Materials and Methods}

Patient Population

Patients referred for elective coronary angiography were candidates for study. Those patients meeting inclusion criteria were approached for participation, and written consent was obtained in accordance with University of New Mexico-Health Sciences Center Human Research Protections Office requirements.
A total of 10 patients provided the sample for this study. Baseline characteristics are shown in Table 1. The intensive analytic requirements and financial cost associated with the processing of each sample are the justification for the feasibility nature of this study. In addition, the magnitude of observed changes at the designated time intervals can be used in power analysis for future study designs.

The non-ionic, iso-osmolar agent, Iodixanol-320 (Visipaque $^{\circledR}$, GE Healthcare), was used in all patients. Blood sampling occurred prior to 4 and $24 \mathrm{~h}$ following RCM. No complications were observed with cardiac catheterization.

In accordance with recommendations by the Human Proteome Organization Plasma Proteome Project [19], plasma samples were analyzed preferentially over serum due to less degradation of the sample ex vivo, and larger numbers of high abundance peptides were present only in serum and not in plasma. P100 tubes from BD (Becton-Dickenson) were used to collect plasma. A sterile blood collection tube was pre-loaded with protease inhibitors, as well as a self-contained system for removing red blood cells and platelets. Samples were collected according to the manufacturer's protocol, placed in $750-\mu \mathrm{L}$ aliquots and stored frozen at $-80^{\circ} \mathrm{C}$ until analysis.

\section{Sample Analysis}

\section{Sample Treatment and iTRAQ Labeling}

The protein expression profiling process was performed in a series of three steps. The samples were first digested and
Fig. 1 Spectral representation of the reproducibility of sample analysis
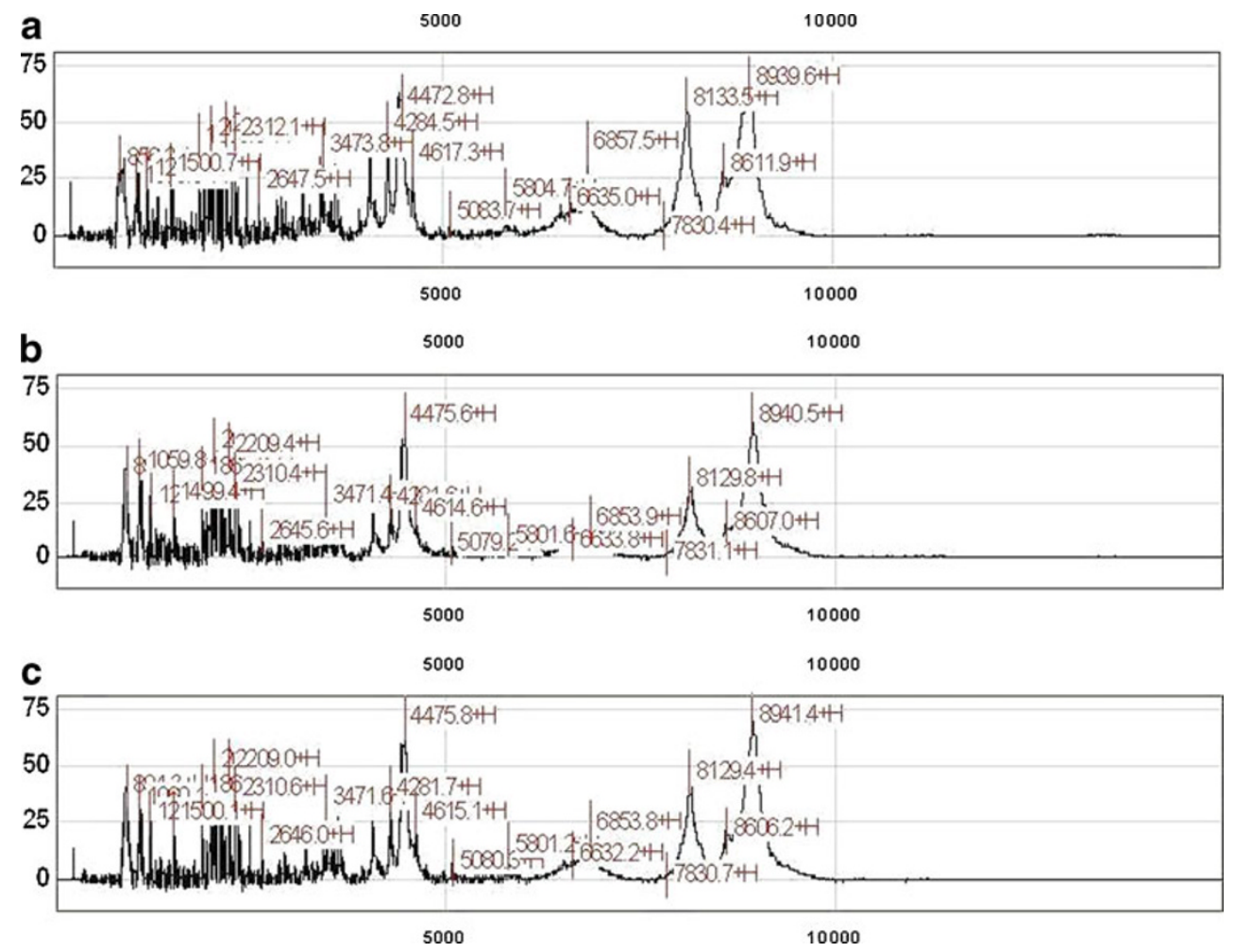
Table 2 All proteins detected with a $\geq 90 \%$ confidence interval (CI)

(O00159) Myosin Ic (myosin I beta; MMI-beta; MMIb)

(O00411) DNA-directed RNA polymerase (MtRPOL)

(O15182) Centrin 3

(O43184) ADAM 12 (A disintegrin and metalloproteinase domain 12)

(O43680) Transcription factor 21 (podocyte-expressed 1; Pod-1; epicardin; capsulin)

(O60241) Brain-specific angiogenesis inhibitor 2

(O60449) Lymphocyte antigen 75 (DEC-205; CD205 antigen; gp200-MR6)

(O60902) Short stature homeobox protein 2 (paired-related homeobox protein SHOT)

(O75427) Leucine-rich repeats and calponin homology domain-containing protein 4 (Leucine-rich neuronal protein)

(O75717) WD repeat and HMG-box DNA-binding protein 1 (acidic nucleoplasmic DNA-binding protein 1 (And-1))

(O96033) Molybdenum cofactor synthesis protein 2 small subunit (molybdopterin-synthase small subunit; MOCS2A; MOCO1-A)

(P00450) Ceruloplasmin (ferroxidase)

(P00734) Prothrombin (coagulation factor II)

(P00738) Haptoglobin

(P01023) Alpha-2-macroglobulin (Alpha-2-M)

(P01024) Complement C3 [contains: C3a anaphylatoxin]

(P01028) Complement C4 [contains: C4a anaphylatoxin; $\mathrm{C} 4 \mathrm{~b}$ ]

(P01266) Thyroglobulin

(P01834) Ig kappa chain $\mathrm{C}$ region

(P01842) Ig lambda chain $\mathrm{C}$ region

(P01871) Ig mu chain $\mathrm{C}$ region

(P01876) Ig alpha-1 chain $\mathrm{C}$ region

(P01877) Ig alpha-2 chain $\mathrm{C}$ region

(P02023) Hemoglobin beta chain

(P02042) Hemoglobin delta chain

(P02647) Apolipoprotein A-I (Apo-AI)

(P02652) Apolipoprotein A-II (Apo-AII; ApoA-II)

(P02671) Fibrinogen alpha/alpha-E chain [contains: fibrinopeptide A]

(P02675) Fibrinogen beta chain [contains: fibrinopeptide B]

(P02743) Serum amyloid P-component (SAP; 9.5S alpha-1-glycoprotein)

(P02748) Complement component C9

(P02751) Fibronectin (FN; cold-insoluble globulin (CIG))

(P02763) Alpha-1-acid glycoprotein 1 (AGP 1; orosomucoid 1; OMD 1)

(P02765) Alpha-2-HS-glycoprotein (fetuin-A; Alpha-2-Z-globulin)

(P02766) Transthyretin (Prealbumin; TBPA; TTR; ATTR)

(P02768) Serum albumin

(P02774) Vitamin D-binding protein (DBP; group-specific component; Gc-globulin; VDB)

(P02787) Serotransferrin (transferrin; siderophilin; Beta-1-metal binding globulin)

(P02790) Hemopexin (beta-1B-glycoprotein)

(P04004) Vitronectin (serum spreading factor; S-protein; V75) [contains: vitronectin V6

(P04196) Histidine-rich glycoprotein (histidine-proline rich glycoprotein (HPRG))

(P04196) Histidine-rich glycoprotein (histidine-proline rich glycoprotein (HPRG))

(P04206) Ig kappa chain V-III region GOL (rheumatoid factor)

(P04217) Alpha-1B-glycoprotein (alpha-1-B glycoprotein)

(P04220) Ig mu heavy chain disease protein (BOT)

(P04264) Keratin, type II cytoskeletal 1 (cytokeratin 1; K1; CK 1; 67-kDa cytokeratin)

(P08912) Muscarinic acetylcholine receptor M5

(P13671) Complement component C6

(P25054) Adenomatous polyposis coli protein (APC protein)

(P25311) Zinc-alpha-2-glycoprotein (Zn-alpha-2-glycoprotein; Zn-alpha-2-GP) 
(P29371) Neuromedin K receptor (NKR; neurokinin B receptor; NK-3 receptor; NK-3R; Tachykinin receptor 3)

(P30307) M-phase inducer phosphatase 3 (dual specificity phosphatase Cdc25C)

(P30968) Gonadotropin-releasing hormone receptor (GnRH receptor; GnRH-R)

(P35789) Zinc finger protein 93 (zinc finger protein HTF34; Fragment)

(P40426) Pre-B-cell leukemia transcription factor-3 (homeobox protein PBX3)

(P49715) CCAAT/enhancer binding protein alpha (C/EBP alpha)

(P56975) Pro-neuregulin-3 (Pro-NRG3) [contains: neuregulin-3 (NRG-3)]

(P56975) Pro-neuregulin-3 (Pro-NRG3) [contains: neuregulin-3 (NRG-3)]

(P57678) Component of gems 4 (Gemin4; p97)

(P61247) 40S ribosomal protein S3a

(Q00975) Voltage-dependent N-type calcium channel alpha-1B subunit

(Q01581) Hydroxymethylglutaryl-CoA synthase, cytoplasmic (HMG-CoA synthase)

(Q08289) Voltage-dependent L-type calcium channel beta-2 subunit (CAB2; calcium channel, voltage-dependent, beta 2 subunit; Lambert-Eaton myasthenic syndrome antigen B; MYSB)

(Q12981) BCL2/adenovirus E1B 19-kDa protein-interacting protein 1

(Q13424) Alpha-1-syntrophin (59-kDa dystrophin-associated protein A1, acidic component 1; Pro-TGF-alpha cytoplasmic domain-interacting protein 1; TACIP1; syntrophin 1)

(Q13772) Nuclear receptor coactivator 4 (NCoA-4; 70-kDa androgen receptor coactivator; 70-kDa AR-activator; Ret-activating protein ELE1)

(Q14624) Inter-alpha-trypsin inhibitor heavy chain H4 (ITI heavy chain H4)

(Q14746) Conserved oligomeric Golgi complex component 2

(Q15697) Zinc finger protein 174 (AW-1)

(Q16288) NT-3 growth factor receptor (TrkC tyrosine kinase; GP145-TrkC)

(Q7RTV0) PHD finger-like domain protein 5A (splicing factor 3B associated 14-kDa protein; SF3b14b)

(Q86V81) THO complex subunit 4 (Tho4; Ally of AML-1 and LEF-1; transcriptional coactivator Aly/REF; bZIP enhancing factor BEF)

(Q8IUC8) Polypeptide $N$-acetylgalactosaminyltransferase 13

(Q8IXZ2) Zinc finger CCCH type domain-containing protein 3

(Q8NCW0) Kremen protein 2 (kringle-containing protein marking the eye and the nose; Dickkopf receptor 2)

(Q8TAC2) Josephin 2 (SBBI54)

(Q8TDZ2) NEDD9 interacting protein with calponin homology and LIM domains (Molecule interacting with CasL protein 1)

(Q8TEX9) Importin 4 (importin 4b; Imp4b; Ran-binding protein 4; RanBP4)

(Q8TEY7) Ubiquitin carboxyl-terminal hydrolase 33 (ubiquitin thiolesterase 33; ubiquitin-specific processing protease 33; deubiquitinating enzyme 33; VHL-interacting deubiquitinating enzyme 1)

(Q8WW01) tRNA-splicing endonuclease subunit SEN15 (tRNA-intron endonuclease SEN15; HsSen15)

(Q8WWL7) G2/mitotic-specific cyclin B3

(Q92575) UBX domain-containing protein 2

(Q96AC1) Pleckstrin homology domain-containing family C member 1 (kindlin 2; mitogen-inducible gene 2 protein; Mig-2)

(Q96T58) Msx2-interacting protein (SMART/HDAC1 associated repressor protein)

(Q99986) Serine/threonine-protein kinase VRK1 (vaccinia-related kinase 1)

(Q9BPX3) Condensin subunit 3 (chromosome-associated protein G; condensin subunit CAP-G; hCAP-G; XCAP-G homolog; NY-MEL-3 antigen)

(Q9BZF1) Oxysterol binding protein-related protein 8 (OSBP-related protein 8 (ORP-8))

(Q9GZQ6) Neuropeptide FF receptor 1 (G protein-coupled receptor 147; RFamide-related peptide receptor OT7T022)

(Q9GZU5) Nyctalopin

(Q9HCU4) Cadherin EGF LAG seven-pass G-type receptor 2 (epidermal growth factor-like 2; multiple epidermal growth factor-like domains 3; flamingo 1)

(Q9NQ38) Serine protease inhibitor Kazal-type 5

(Q9NSI6) WD-repeat protein 9

(Q9NZM3) Intersectin 2 (SH3 domain-containing protein 1B; SH3P18; SH3P18-like WASP associated protein)

(Q9Y282) Serologically defined breast cancer antigen NY-BR-84 (CGI-54)

(Q9Y2K5) R3H domain protein KIAA1002 (fragment)

(Q9Y2Q0) Potential phospholipid-transporting ATPase IA (chromaffin granule ATPase II)

(Q9Y305) Putative acyl-CoA thioester hydrolase CGI-16

(Q9Y5A7) NEDD8 ultimate buster-1 (NY-REN-18 antigen) 
(Q9Y6D6) Brefeldin A-inhibited guanine nucleotide-exchange protein 1 (brefeldin A-inhibited GEP 1; p200 ARF-GEP1; p200 ARF guanine nucleotide exchange factor)

(Q9Y6K8) Adenylate kinase isoenzyme 5 (ATP-AMP transphosphorylase)

(Q9Y6N9) Harmonin (Usher syndrome 1C protein; Autoimmune enteropathy-related antigen AIE-75; Antigen NY-CO-38/NY-CO-37; PDZ-73 protein)

(Q9Y6X9) Zinc finger CW-type coiled-coil domain protein 1

labeled using an Applied Biosystems iTRAQ labeling protocol, then placed through a cleanup and prefractionation step, and finally placed through an mass spectrometry (MS)/MS analysis on the Applied Biosystems matrix-assisted laser desorption/ionization (MALDI) 4700. The iTRAQ kit, from Applied Biosystems, allowed multiple samples to be processed and analyzed concurrently for direct comparison of the results.

Each sample was removed from the $-80^{\circ} \mathrm{C}$ freezer and delipidated according to the chloroform method as previously reported [20], with removal of $60 \%$ of lipids from human plasma samples. The samples were then desalted using the Zebra Desalt Spin columns from Pierce, a process which removes $>95 \%$ of the salts and small molecules under 1,000 Da from the plasma sample. The samples are then frozen overnight and labeled the following day. After delipidation and desalting protein concentrations in the samples were measured by the Bradford assay (Bio-Rad Laboratories). Of each plasma sample time point, $100 \mu \mathrm{g}$ was processed according to the iTRAQ manufacturer's instruction (Applied Biosystems). The iTRAQ labeling protocol incorporates the following: a dissolution buffer, to dissolve the proteins and buffer the subsequent reactions; a denaturant, which disrupts the hydrogen, hydrophobic and electrostatic bonds; a reducing reagent, which reduces the disulfide bonds; and a cysteine blocking reagent, which reversibly blocks all cysteine residues. The denatured proteins are then digested with trypsin and labeled with the iTRAQ mass tags. For each individual patient, plasma was collected at three different time points. The first time point was labeled with the iTRAQ tag of mass 114, the second time point with tag 115 , and the third time point with tag 116. After labeling all three time points, samples are combined into a single patient sample. After the samples are labeled, they are cleaned and fractionated through a twodimensional high-performance liquid chromatography (HPLC) separation.

\section{Cation Exchange Chromatography}

The combined peptide mixture is separated by strong cation exchange (SCX) chromatography on a Shimadzu 10VP HPLC system using a PolySulfoethyl A column $(4.6 \times$ $100 \mathrm{~mm}, 5 \mu \mathrm{m}, 300 \AA$ A, PolyLC, Columbia, MD). The sample in the SCX exchange buffer is loaded and washed isocratically for $20 \mathrm{~min}$ at $0.5 \mathrm{ml} / \mathrm{min}$ to remove excess iTRAQ reagents. Peptides are eluted with a linear gradient of 0-500 mM KCl (25\% v/v ACN, $\left.10 \mathrm{mM} \mathrm{KH}_{2} \mathrm{PO}_{4}, \mathrm{pH} 3\right)$ over $20 \mathrm{~min}$ at a flow rate of $1 \mathrm{ml} / \mathrm{min}$, with $1-\mathrm{ml}$ fractions collected at room temperature. SCX fractions are dried down completely to reduce volume, then resuspended in $2 \%(v / v)$ acetonitrile, $0.1 \%(v / v)$ trifluoroacetic acid, and filtered prior to reverse phase $\mathrm{C} 18$ nanoflow-LC separation. The fractions are then taken and separated on a $\mathrm{C} 18$ column by peptide mass.

\section{LC-MS Analysis}

Peptide separation is performed on an Ultimate chromatography system (Dionex-LC Packings, Hercules, CA) equipped with a Probot MALDI spotting device. Individual SCX fractions containing $10 \mu \mathrm{g}$ of protein material are injected and captured onto a $0.3 \times 5-\mathrm{mm}$ trap column $(3-\mu \mathrm{m}$ $\mathrm{C}_{18}$ (Dionex-LC Packings, Hercules, CA)) and then eluted onto a $75 \mu \mathrm{m} \times 15 \mathrm{~cm}$ Pepmap analytical column $\left(5-\mu \mathrm{m} \mathrm{C}_{18}\right.$ (Dionex-LC Packings)) using a binary gradient $(200 \mathrm{nl} /$ min) from $5 \%$ to $45 \%$ buffer B with mobile phase A $2 \%$ ACN, 0.1\% TFA, and mobile phase B 85\% ACN, 5\% isopropanol, $0.1 \%$ TFA). For MALDI MS/MS analysis, column effluent is mixed in a 1:2 ratio with MALDI matrix (7 mg/ml cyano-4-hydroxycinnamic acid) through a 25-nl mixing tee (Upchurch Scientific, Oak Harbor, WA) and spotted in 192 spot arrays. Finally, the MALDI plates are placed in the Applied Biosystems 4700 MALDI-time-offlight (TOF) and are analyzed in MS/MS mode.

\section{Surface Enhanced Laser Desorption/Ionization-TOF MS Profiles}

Samples were initially screened using Ciphergen's surface enhanced laser desorption/ionization (SELDI) MS technology. Protein profiles were obtained using a PBSIIc ProteinChip reader (Ciphergen). Before analysis, the instrument was externally calibrated using all-in-one peptide standard (Ciphergen) using a linear calibration equation. High mass was set to $15,000 \mathrm{Da}$, optimizing between 1,000 and 10,000 Da. Detector sensitivity was set to 9, laser intensity to 180 , and mass deflector to 3,000 Da. Two warming shots fired at each new position at laser intensity 210 were not included in the profile. The source voltage and detector voltage was at 20,000 and 3,000 V, respec- 
Table 3 Proteins common to all 10 patients

Proteins

Number of patients with identified protein

(P01023) Alpha-2-macroglobulin (Alpha-2-M)

(P01024) Complement C3 [contains: C3a anaphylatoxin]

(P02647) Apolipoprotein A-I (Apo-AI)

(P02768) Serum albumin

(P02787) Serotransferrin (transferrin; siderophilin; beta-1-metal binding globulin)

(P02790) Hemopexin (beta-1B-glycoprotein)

(P00738) Haptoglobin

(P02652) Apolipoprotein A-II (Apo-AII; ApoA-II)

(O75717) WD repeat and HMG-box DNA-binding protein 1 (acidic nucleoplasmic DNA-binding protein 1

(And-1))

(P00450) Ceruloplasmin (ferroxidase)

(P02763) Alpha-1-acid glycoprotein 1 (AGP 1; orosomucoid 1; OMD 1)

(P02766) Transthyretin (prealbumin; TBPA; TTR; ATTR)

(P02774) Vitamin D-binding protein (DBP; group-specific component; Gc-globulin; VDB)

(P30307) M-phase inducer phosphatase 3 (dual specificity phosphatase Cdc25C)

(Q14746) Conserved oligomeric Golgi complex component 2

(P01877) Ig alpha-2 chain C region

(O96033) Molybdenum cofactor synthesis protein 2 small subunit (molybdopterin-synthase small subunit;

MOCS2A; MOCO1-A)

(P49715) CCAAT/enhancer binding protein alpha (C/EBP alpha)

(Q8WW01) tRNA-splicing endonuclease subunit SEN15 (tRNA-intron endonuclease SEN15; HsSen15)

(Q9Y6D6) Brefeldin A-inhibited guanine nucleotide-exchange protein 1 (brefeldin A-inhibited GEP 1; p200 ARF-GEP1; p200 ARF guanine nucleotide exchange factor)

(P00734) Prothrombin (coagulation factor II)

(P01028) Complement C4 [contains: C4a anaphylatoxin; C4b]

(P02675) Fibrinogen beta chain [contains: fibrinopeptide B]

(P29371) Neuromedin K receptor (NKR; neurokinin B receptor; NK-3 receptor; NK-3R; tachykinin receptor 3)

(P57678) Component of gems 4 (Gemin4; p97)

(Q13424) Alpha-1-syntrophin (59-kDa dystrophin-associated protein A1, acidic component 1; Pro-TGF-alpha

(Q9NQ38) Serine protease inhibitor Kazal-type 5

(O43680) Transcription factor 21 (podocyte-expressed 1; Pod-1; epicardin; capsulin)

(P02671) Fibrinogen alpha/alpha-E chain [contains: fibrinopeptide A]

(P02743) Serum amyloid P-component (SAP; 9.5S alpha-1-glycoprotein)

(P02748) Complement component C9

(P04196) Histidine-rich glycoprotein (histidine-proline rich glycoprotein; HPRG)

(P04220) Ig mu heavy chain disease protein (BOT)

(Q00975) Voltage-dependent N-type calcium channel alpha-1B subunit

(Q8TEY7) Ubiquitin carboxyl-terminal hydrolase 33 (ubiquitin thiolesterase 33; ubiquitin-specific processing protease 33; deubiquitinating enzyme 33; VHL-interacting deubiquitinating enzyme 1) (Q96T58) Msx2-interacting protein (SMART/HDAC1 associated repressor protein)

(Q99986) Serine/threonine-protein kinase VRK1 (vaccinia-related kinase 1) 

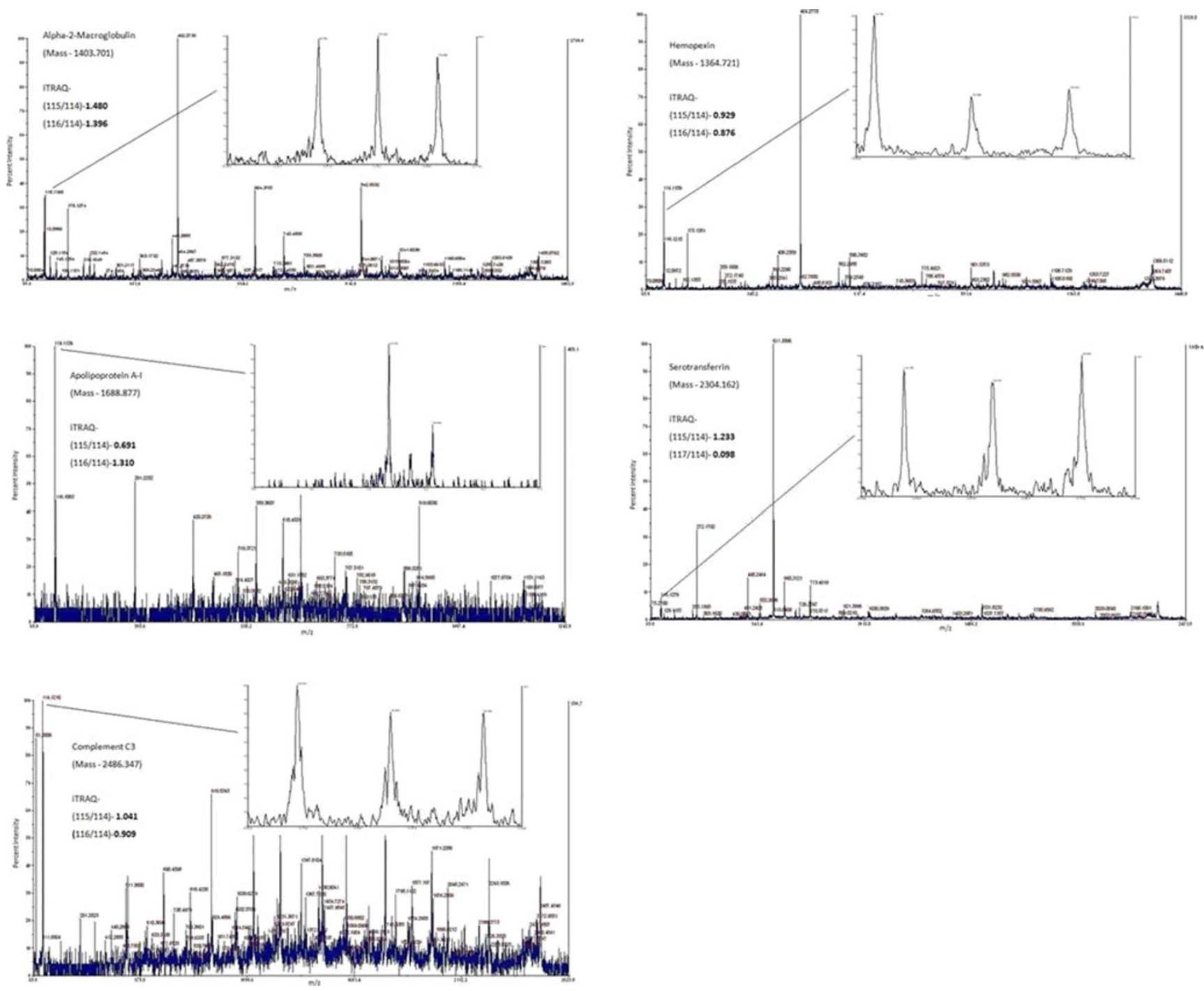

Fig. 2 Mass spectra of the proteins identified in this patient population. The outset spectra show the plasma sample averaged for all time-points. The inset spectra show the individual time-points

tively. The samples were run in triplicate using the CM10 (Ciphergen, Fremont, CA) weak cation exchange protein chip according to the manufacturer's protocol, as shown in Fig. 1.

\section{Tandem Mass Spectrometry}

All MS and MS/MS spectra are acquired on an Applied Biosystems 4700 Proteomics Analyzer (TOF/TOF; Applied Biosystems/MDX Sciex, Foster City, CA) in positive ion reflection mode. MS survey scans are acquired for each sample across the entire plate from $\mathrm{m} / \mathrm{z}$ ranges 500-4,000. All peaks above an $S / N$ of 60 are selected for MS/MS analysis.

\section{Protein Identification and Analysis}

Both MS and MS/MS spectra are processed in GPS ExplorerTM software (v3.5, Applied Biosystems). For MS spectra, an
$S / N$ threshold of 30 was used; for MS/MS spectra, a threshold of 20 was used to detect peaks. Monoisotopic peak lists were generated in GPS Explorer and submitted to the Mascot search tool for protein identities. For all searches, ion mass tolerance was $100 \mathrm{ppm}$, and fragment ion mass tolerance was 0.6 Da. Protein identification was considered significant with a Mascot score corresponding to $p<0.05$. The GPS software prepares a list of identified peptides with reporter ion peak average ratios and standard deviations. A statistical calculation of how closely the acquired data matches previous database searches under differing conditions. This calculation uses normal probability distribution mathematics, also used by the Mascot search engine for scoring. Because different database searches have different Mascot significance levels, protein scores and ion scores from different searches cannot be directly compared. Different Mascot significance levels are obtained if databases of different sizes are searched or if 
different numbers of masses are submitted for a database search. Therefore, the confidence interval (CI) is calculated from the Mascot protein score or ion score, but the significance level is removed from the calculation to allow comparison between results from different database searches. In our case, ion score was used to show significance. CI rates the confidence level of the protein score or ion score. If the score returned from the search is equal to the Mascot significance level for the search, the score is given a $95 \%$ confidence interval. Scores that are higher or lower than the Mascot significance level for the search are given higher or lower confidence intervals, respectively. The closer the $\mathrm{CI} \%$ is to $100 \%$, the more likely the protein is correctly identified. Of note, if the calculated $\mathrm{CI}>100$, then 100 is reported, and if $<0$, then 0 is reported.

A semi-quantitative protein analysis on proteins of interest was performed using their relative iTRAQ ratios. The protein iTRAQ ratios were compared using their relative baseline, 4- and 24-h levels using a one sample $t$ test. A one sample $t$ test was used for comparison due to sole interest in deviation (either increase or decrease) from its baseline level.

\section{Results}

A total of 102 proteins were identified ( $\mathrm{CI} \geq 90 \%)$, based on ion score, as shown in Table 2. Of the proteins with a CI $\geq 90 \% \quad(n=102), 42$ total proteins were identified in $\geq 2$ patients, and 16 proteins (with a $\geq 90 \% \mathrm{CI}$ ) were identified in the majority of patients (Table 3 ). Of these 16 proteins, six proteins were present in all patients: serum albumin, alpha-2-macroglobulin, apolipoprotein A-I, complement C3, hemopexin, and serotransferrin. Representative spectra for these common proteins are depicted in Fig. 2. Due to the known significant abundance of serum albumin, its parent protein was not further analyzed.

iTRAQ studies yield a differential comparison of protein expression with reference to a control state. In these studies, each patient's baseline sample served as their control state. Although absolute amounts of proteins may vary between individuals, it is the relative change that was assessed. Individual quantification of a peptide is based on the ratios of the ITRAQ signature ions (as shown in Fig. 2). The protein iTRAQ ratios are determined by averaging all of the ratios for all of the peptides found for each protein. Levels of nearly all proteins identified were found to decrease (iTRAQ $<1)$ at $4 \mathrm{~h}$ compared to baseline with a return to baseline or near baseline at $24 \mathrm{~h}$ (iTRAQ $\geq 1$ ). Of interest, a near consistent consumptive process was noted in identified proteins recognized in the immune, thrombotic, hematological, and lipoprotein physiologic processes at $4 \mathrm{~h}$ following administration of RCM that trended toward baseline values at $24 \mathrm{~h}$, as shown in Table 4 .

\section{Discussion}

High-throughput proteomic analysis of patients undergoing elective coronary angiography identified 102 distinct proteins in this sample of 10 patients. These proteins were reliably identified with $\geq 90 \%$ CI. At this level of confidence, 42 proteins were noted to be common among patients and involved in various crucial physiologic processes. Sixteen proteins were identified in the majority of patients. Six proteins were present in all patients and included serum albumin, apolipoprotein A-I, hemopexin, serotransferrin, alpha-2-macroglobulin, and complement C3. Interestingly, when analyzing the proteins pertaining to the immune, thrombotic, hematological, and lipoprotein physiologic processes, iTRAQ ratios revealed a consumptive process during the 4-h sampling that tended to return to baseline by $24 \mathrm{~h}$. Overall, these observations suggest that administration of RCM results in significant changes in the proteome and that there is activation of hematologic, inflammatory, pro-thrombotic, and lipid transport processes.

Prior studies on patients with chronic coronary artery disease have shown that RCM can act as a potent inducer of

Table 4 Proteins identified in patients with given iTRAQ ratios comparing baseline to 4 and $24 \mathrm{~h}$

\begin{tabular}{lllllcccrrr}
\hline & $\begin{array}{l}\text { Alpha-2- } \\
\text { MG }\end{array}$ & $\begin{array}{l}\text { Comp } \\
\text { C3 }\end{array}$ & $\begin{array}{l}\text { Apo- } \\
\text { AI }\end{array}$ & $\begin{array}{l}\text { Apo- } \\
\text { AII }\end{array}$ & Serotransferrin & Hemopexin & $\begin{array}{l}\text { Fibrinogen beta } \\
\text { chain }\end{array}$ & $\begin{array}{l}\text { Prothrombin } \\
\text { Comp } \\
\text { C4 }\end{array}$ & $\begin{array}{l}\text { Ig } \\
\text { alpha }\end{array}$ \\
\hline iTRAQ4h & 0.78 & 0.89 & 0.61 & 0.70 & 0.86 & 0.93 & 0.63 & 0.60 & 0.32 & 0.70 \\
iTRAQ24h & 1.03 & 1.24 & 0.86 & 0.87 & 0.96 & 1.11 & 0.85 & 0.58 & 1.00 & 0.86 \\
$P$ value & 0.004 & 0.02 & $<0.001$ & $<0.001$ & $<0.001$ & 0.0012 & 0.01 & 0.04 & $>0.05$ & $<0.001$ \\
$\begin{array}{l}4 \quad \mathrm{~h} \\
P \text { value }\end{array}$ & 0.01 & $>0.05$ & $<0.001$ & 0.02 & $<0.001$ & 0.03 & $>0.05$ & $>0.05$ & $>0.05$ & $<0.001$ \\
$24 \mathrm{~h}$ & & & & & & & & & \\
\hline
\end{tabular}

Alpha-2-MG alpha-2-macroglobulin, Comp C3 complement C3, Apo-AI apolipoprotein A-I, Apo-AII apolipoprotein A-II, Comp C4 complement $\mathrm{C} 4$, Ig alpha immunoglobulin alpha-2 chain $\mathrm{C}$ region, $P$ value $4 h p$ value comparing baseline to 4-h iTRAQ ratios, $P$ value $24 h p$ value comparing baseline to 24-h iTRAQ ratios, $i T R A Q 4 h$ and $i T R A Q 24 h$ rate of protein species at 4 and $24 \mathrm{~h}$, respectively, compared to baseline 
the inflammatory pathway with increases in C-reactive protein, interleukin-6, tumor necrosis factor-alpha, and complement components $[12,13]$. Some studies by others have attributed pro-thrombotic potential to RCM [16], an observation thought to be more related to ionic properties of RCM [9]. In vitro studies have also revealed RCM to be an inducer of platelet degranulation, which has been implicated as a contributor to the thrombotic potential of RCM [3], a finding that has been refuted by in vivo analysis [5]. Additive evidence also exists for an injurious effect on vascular endothelium with administration of RCM [21].

The value of the approach utilized herein is the demonstration that multiple pathways and protein responses can be studied in each patient and that, using each patient as their own control, dynamic changes over time can be studied. The demonstration of simultaneous activation of inflammatory and thrombotic pathways consequent to RCM administration provides incentive for further study in patients at risk of thrombotic events. Furthermore, this approach allows for comparative analysis among the different types of RCM.

In summary, the complex and dynamic changes in the human proteome consequent to RCM administration provide further insight into mechanisms of potential RCMassociated adverse clinical effects. The observations, herein, of alterations in inflammatory-, thrombotic-, and lipoprotein-related processes require further study in large populations to better define the clinical relevance of these observations.

Acknowledgments This study has been supported in part by the Robert S. Flinn Endowment for Cardiovascular Medicine (University of New Mexico School of Medicine) and GE Healthcare, Ltd. We thank Melissa Candelaria-Lyons and Lindsay Candelaria for their significant assistance in sample analysis and data generation.

\section{References}

1. Lloyd-Jones D, Adams RJ, Brown TM, American Heart Association Statistics Committee and Stroke Statistics Subcommittee, et al. Heart disease and stroke statistics-2010 update: a report from the American Heart Association. Circulation. 2010;121:e1-e170.

2. Holmes JS, Kozak LJ, Owings MF. Use and in-hospital mortality associated with two cardiac procedures, by sex and age: national trends, 1990-2004. Health Affairs (Millwood). 2007;26:169-77.

3. Chronos NA, Goodall AH, Wilson DJ, Sigwart U, Buller NP. Profound platelet degranulation is an important side effect of some types of contrast media used in interventional cardiology. Circulation. 1993;88:2035-44.

4. Grabowski EF, Jang IK, Gold H, Head C, Benoit SE, Michelson AD. Variability of platelet degranulation by different contrast media. Acad Radiol. 1996;3:S485-7.
5. Sakariassen KS, Barstad RM, Hamers MJ, Stormorken H. Platelet activation and thrombosis. I. Iohexol-induced platelet secretion does not affect thrombus formation in native blood. Acta Radiol. 1998;39:349-54.

6. Sakariassen KS, Barstad RM, Hamers MJ, Stormorken H. Platelet activation and thrombosis. II. Iohexol-induced platelet secretion does not affect collagen-induced or tissue-factor induced thrombus formation in blood that is anticoagulated with heparin and aspirin. Acta Radiol. 1998;39:355-61.

7. Rasmussen F. The influence of radiographic contrast media on some granulocyte functions. Acta Radiol. 1998;39:7-35.

8. Rasmussen F. Radiographic contrast media and release of neutrophil specific proteins in vitro and after intravenous injection. Acta Radiol. 1992;33:495-9.

9. Owens MR, Ribes JA, Marder VJ, Francis CW. Effects of ionic and nonionic radiographic contrast agents on endothelial cells in vitro. J Lab Clin Med. 1992;119:315-9.

10. Riemann CD, Massey CV, McCarron DL, Borkowski P, Johnson $\mathrm{PC}$, Ziskind AA. Ionic contrast agent mediated endothelial injury causes increased platelet deposition to vascular surfaces. Am Heart J. 1993;125:71-8.

11. Davidson CJ, Laskey WK, Hermiller JB, et al. Randomized trial of contrast media utilization in high-risk PTCA: the COURT trial. Circulation. 2000;101:2172-7.

12. Bertrand ME, Esplugas E, Piessens J, Rasch W. Influence of a nonionic, iso-osmolar contrast medium (iodixanol) versus an ionic, low-osmolar contrast medium (ioxaglate) on major adverse cardiac events in patients undergoing percutaneous transluminal coronary angioplasty: a multicenter, randomized, double-blind study. Circulation. 2000;101:131-6.

13. Laskey WK, Gellman J. Inflammatory markers increase following exposure to radiographic contrast media. Acta Radiol. 2003;44:498-503.

14. Goldberg A, Zinder O, Zdorovyak A, et al. Diagnostic coronary angiography induces a systemic inflammatory response in patients with stable angina. Am Heart J. 2003;146:819-23.

15. Deftereos S, Giannopoulos G, Kossyvakis C, et al. Effects of radiographic contrast media on markers of complement activation and apoptosis in patients with chronic coronary artery disease undergoing coronary angiography. J Invasive Cardiol. 2009;21:473-7.

16. Fauser C, Ullisch EV, Kubler W, Haller C. Differential effects of radiocontrast agents on human umbilical vein endothelial cells: cytotoxicity and modulators of thrombogenicity. Eur J Med Res. 2001;11:465-72.

17. Robertson HJ. Blood clot formation in angiographic syringes containing nonionic contrast media. Radiology. 1987;162:621-2.

18. Grollman Jr JH, Liu CK, Astone RA, Lurie MD. Thromboembolic complications in coronary angiography associated with the use of nonionic contrast media. Cathet Cardiovasc Diagn. 1988;14:159 64.

19. Omenn GS, States DJ, Adamski M, et al. Overview of the HUPO Plasma Proteome Project: results from the pilot phase with 35 collaborating laboratories and multiple analytical groups, generating a core dataset of 3020 proteins and a publicly-available database. Proteomics. 2005;13:3226-45.

20. Castro AR, Morrill WE, Pope V. Lipid removal from human serum samples. Clin Diagn Lab Immunol. 2000;7:197-9.

21. Nyman U, Almen T. Effects of contrast media on aortic endothelium. Experiments in the rat with non-ionic monomeric and monoacidic dimer contrast media. Acta Radiol Suppl. 1980;362:65-71. 